Temática

\title{
La Negociación con el Estado: Las Tácticas de las Mujeres SAUDITAS
}

\author{
Alejandra Galindo Marines
}

Resumen: El principal objetivo de este artículoes comprender cómo algunas mujeres reclaman sus derechos para gozar de la ciudadanía. Con el análisis de las tácticas usadas por las mujeres en años recientes, buscamos esclarecer cómo la mujer ha aprovechado dos momentos históricos, la invasión de Irak a Kuwait (1990-1991) y los eventos relacionados con el 11 de septiembre, para que su voz sea escuchada por la sociedad y el gobierno. Nosotros afirmamos que estas mujeres no solamentenegocian en la esfera privada, sino también negocian con el Estado en términos de ciudadanas, apropiándose y transformando los símbolos tradicionales y las representaciones que actualmente están vigentes. Este artículo nos permitirá reconocer que las concepciones occidentalizadas de ciudadanía fallan al no tomar en cuenta los matices y particularidades de los países en desarrolloy particularmente de Arabia Saudita.

Palabras clave: Arabia Saudita, Género, Ciudadanía, Mujeres.

Enviado a dictamen: 18 de agosto de 2008.

Aprobación: 29 de octubre de 2008.

Dra. Alejandra Galindo Marines, Doctora en Política de Medio Oriente, Durham University, G.B., Profesora Titular del Departamento de Ciencias Sociales, Universidad de Monterrey, temas de especialización: La problemática de la relación entre religión y política desde el Estado-sociedad, así como también las relaciones internacionales del Golfo Árabe, correo electrónico: agalindo@udem.edu.mx.
Abstract: The main goal of this article is to understand how some women claim their rights to enjoy full citizenship. By examining the tactics of women in recent years, we aim to elucidate on how women have taken advantage of two historic moments, Iraq invasion to Kuwait (1990-1991) and the events around 9/11, in order to be heard by society and government. We contend that not only women negotiate in the private sphere, since they also negotiate with the state in terms of citizens, subverting the traditional symbols and representations currently at place. The article aims to acknowledge that westernized conceptions of citizenship fail to grasp the nuances and particularities of underdeveloped countries and specifically Saudi Arabia.

Key words: Saudi Arabia, Sort, Citizenship, Women.

I

nvestigar sobre género y ciudadanía en una sociedad no occidental y en desarrollo plantea varias cuesLiones importantes desde el punto de vista teóricometodológico. ${ }^{1}$ La concepción y práctica de ciudadanía en occidente, presenta un patrón excepcional cuando se compara con otras sociedades. En los casos de las sociedades en desarrollo la ciudadanía está atrapada en las estrategias de los estados "modernos" de crear una nación y una identidad nacional. Para las sociedades no occidentales, el aparato colonial y luego los estados in- 
dependientes incursionaron en la vida de los individuos, antes que ellos se pudieran identificar como una nación $y$ antes que les fuera otorgado el estatus de ciudadanos, bajo las constituciones de sus países (Chatherjee, 2006: 1-25). El concepto de ciudadanía comprende tres siglos de desarrollo para el mundo occidental, mientras que en los Estados de creación relativamente reciente, el acceso a los derechos es una lucha que se está enfrentando, especialmente cuando se refiere a las mujeres.

La noción universal y liberal de la ciudadanía se evidencia en las sociedades en desarrollo, al no corresponder a las bases individuales que se encuentran en ésta, ni al papel del Estado como garante del goce de los derechos individuales de los ciudadanos. La ciudadanía en el contexto de las sociedades no occidentales, contrario a la noción liberal, no está ligada al individuo por sí mismo, sino el individuo en función de su pertenencia a la familia y/o grupo étnico/religioso, lo cual en las sociedades del Medio Oriente se entreteje con las características patriarcales de los estados en cuestión. En los países del Medio Oriente es notoria la influencia del patriarcado y de las relaciones de parentesco a través de los hombres, pues constituyen el filtro primario para la constitución de las identidades, naciones y Estados, del mismo modo que la religión (Joseph, 2000:17). En este sentido retomando a Yuval-Davies, el espacio de la familia y las relaciones de parentesco nos indican que la separación entre esfera privada y pública, como base de la constitución del Estado moderno, no necesariamente se encuentra tan claramente dividida (Yuval-Davies, 1997:14).

En el caso de las sociedades no desarrolladas, como en el caso del Medio Oriente, un elemento cultural que influye en la dinámica del proceso de modernización es el papel de la religión. Para algunos como Niblock (1981) y Piscatori (1996), los procesos de modernización en estas sociedades trajeron como consecuencia la consolidación de formas de autoridad tradicionales, como lo muestra el caso de Arabia Saudita. De este modo, la realidad "moderna" coexiste con otras realidades premodernas y en algunos casos postmodernas. El Islam es un parámetro de la política, sociedad y cultura en los diferentes sistemas políticos del Medio Oriente, pero su influencia varía de acuerdo a los términos que cada régimen establezca, así como a los tipos de prácticas de las mismas sociedades, por ello es que no hay un Islam monolítico, sino diferentes tipos de Islam de acuerdo con las sociedades donde se practica y con la especificidad histórica.

Tomando en cuenta estos factores se puede decir que a pesar de la existencia de variables independientes en cada realidad social y cultural, y pese a la genealogía occidental de la concepción de ciudadanía, hay un conjunto de derechos y obligaciones derivados de la membresía a un Estado. Retomando la propuesta de Turner, la ciudadanía cubre los derechos y obligaciones en el aspecto legal, político y social (Turner, 2000: 32-33). Sin embargo, el proceso de definición de ciudadanía se encuentra asociado con un acceso a recursos escasos controlado por el Estado (Turner, 2000: 38) y la asignación de estos recursos está mediada por las percepciones de género dentro de la sociedad, al igual que el género es un espacio para la articulación de poder (Scott, 10671069). Por lo tanto, género y ciudadanía son dos concepciones que están basadas en procesos de inclusión y exclusión, al mismo tiempo que son parte y efecto de las articulaciones de poder. La pertenencia a determinado sexo, grupo étnico, clase social, la proximidad al centro de poder y el papel de la religión son los factores que moldean el ejercicio de la ciudadanía.

Este artículo tiene como finalidad analizar cómo las mujeres sauditas desarrollan sus propias tácticas de demandas para su reconocimiento y participación en la sociedad frente a un Estado que les limita el ejercicio de la ciudadanía, debido a las características patriarcales y de parentesco imbricadas en el reino saudita y a las condiciones que limitan la organización ciudadana. Lo anterior, en contraste con otras sociedades del Medio Oriente, es un fenómeno relativamente nuevo, ya que las mujeres a partir de los noventa, con la estrategia de liberalización del Estado saudita, se empiezan a organizar 
para reclamar una concepción y práctica incluyente de ciudadanía. Si bien existen diferentes voces dentro de las mujeres sauditas, pues hay grupos que reclaman un "status quo"de las mujeres bajo una visión tradicional amparada en los discursos e interpretaciones de los ulema oficiales, al igual que voces reformistas dentro del campo religioso que re-interpretan los significados; no todas han adoptado una postura pública y han confrontado al Estado. ${ }^{2}$

Este artículo se centra en el grupo de mujeres que ha participado en la elaboración de la petición para su reconocimiento y/o en prácticas que cuestionan los roles adscritos a las mujeres en el contexto de la sociedad saudita en la esfera pública. Las tácticas del débil se basan principalmente en la utilización del tiempo para sacudir los cimientos del poder (DeCerteau, 1996: 43-45). En este sentido las mujeres aprovechan los intersticios del poder para hacerse visibles ante el Estado y la sociedad, al ocasionar en algunos casos la negociación con el Estado y los diferentes grupos de la sociedad y al aprovecharse de los momentos coyunturales oportunos.

Nuestro análisis se centrará en dos momentos históricos importantes para el Reino de Arabia Saudita. El primero dado a partir de la crisis ocasionada por la invasión de Irak a Kuwait (1990-1991), así como la aparición pública de demandas por parte de grupos opositores. El segundo comprende los años del nuevo milenio a partir de los eventos del 11 de septiembre, el contexto regional y el resurgimiento de voces opositoras dentro del Reino. Las mujeres sauditas en un primer momento buscan la realización de intereses prácticos, es decir la satisfacción de las necesidades que surgen del emplazamiento de las mujeres dentro de la división sexual del trabajo (Molyneux, 2003: 237). En el segundo momento, cuando el Estado y sociedad debaten sobre la relación gobernantes-gobernados, un grupo de mujeres logra articular una serie de demandas que se identifican con los intereses estratégicos, los cuales representan reivindicaciones de transformación de la sociedad con la finalidad de dar una nueva posición a "la mujer" dentro de las relaciones de género y sociales en general (Molyneux, ibid.).

El elemento central de sus tácticas es apropiarse del discurso para poder cambiar los significados y prácticas adscritas a las mujeres en Arabia Saudita. Las tácticas adoptadas por las mujeres van desde las reuniones y agrupaciones que forman para proveerse de espacios propios y a través de ellos identificar intereses que promuevan una participación de las mujeres. Aceptamos que su acceso a los recursos materiales y culturales está dado en primera instancia por la negociación en la esfera privada, donde en su calidad moral de madres y educadoras promueven un cambio de relaciones familiares, el cual podrá eventualmente incidir en cambios de la esfera pública (Altorki, 2000: 324-325). Nuestro análisis sostiene que el tipo de negociación de algunas mujeres con el Estado no se realiza necesariamente a partir de sus roles tradicionales. Al contrario, las mujeres han reclamado sus derechos y reconocimiento del Estado en su condición de ciudadanas y participantes en la defensa y construcción del futuro del reino saudita. Como señala Dietz, los valores que tienen que defender las mujeres no son tanto los maternales, como los políticos (la libertad, igualdad, el poder comunitario), porque sólo cuando estos valores políticos han sido asumidos, se podrá trabajar en asuntos específicos, como los maternales (Dietz, 1994: 65).

La respuesta del Estado a sus demandas está en relación con la naturaleza de las coyunturas y sus propias necesidades de legitimidad y supervivencia. Si bien el Estado dentro de la narrativa de modernización de los países del Medio Oriente puede verse como un agente del desarrollo, en la práctica su actuación es más bien contradictoria. Por una parte promueve el desarrollo de las mujeres, pero por otra lo circunscribe al ámbito de las prácticas sociales y religiosas, promoviendo el avance de la mujer en su calidad de educadora de los ciudadanos del país. Sin embargo el acceso de la mujer a la educación, salud, empleo constituye en sí mismo una manera de ingresar a las mujeres en la esfera pú- 
blica (Abu-Lughod, 2002: 32). Aunque como advierte Hatem, uno tiene que ser precavido con la narrativa de la modernización y el papel del Estado, pues no necesariamente el ingreso a la esfera pública en estos términos significa una progresión lineal hacia la obtención de una ciudadanía plena. La obtención de los derechos en calidad de ciudadanas como lo muestra el caso de Turquía y Egipto en sus etapas nacionalistas, no necesariamente permanece, pues al instaurarse nuevos regímenes en estos países los cambios introducidos sufrieron un "retroceso" y limitaron la autonomía de los movimientos femeninos (Hatem, 2002: 63-82). Por lo tanto, la obtención de los derechos no siempre es lineal, también puede existir retrocesos y limitaciones.

La primera parte señalará las características principales del Estado Saudita en función de los elementos que están presentes en el ejercicio de la ciudadanía y la representación de la mujer considerados desde el punto de vista del Estado y la sociedad. Una vez analizadas las estructuras y prácticas de subordinación de la mujer se procederá a examinar el contexto y la formulación de la participación pública de las mujeres en su manifestación. La segunda parte de nuestro artículo abordará el contexto en el cual surgen las demandas y participación pública, al analizar primeramente cómo negocian las mujeres activistas (aquellas que abogan por la presencia femenina y participan en los diferentes momentos) en la esfera privada, para después señalar los diferentes tipos de organización de mujeres a partir de la década de los noventa. Lo anterior nos permitirá entender cómo las mujeres van tejiendo redes para nuevamente entrar en el ámbito público en dos momentos: el primero como participantes en el debate sobre la defensa de la nación; y, luego, en sus intentos por participar en las elecciones municipales y en los consejos de las cámaras de comercio. Finalmente reflexionaremos sobre las tácticas que emplean las mujeres en su lucha por la redefinición de la ciudadanía. La base de este artículo es principalmente una investigación de campo realizada en Riyad, Damam, Al Jobar y Jedda en Arabia Saudita (diciembre 2006-marzo 2007) con mujeres ${ }^{3}$ que participan desde diferentes ámbitos en la construcción de una ciudadanía activa de las mujeres, así como también entrevistas realizadas a líderes de la oposición exiliados y entrevistas realizadas en Riyad en 1999 como parte de mi primera estancia en el país.

\section{La construcción de la ciudadanía}

En esta sección nos interesa destacar cómo el Estado, de acuerdo con sus necesidades de legitimidad, construye estructuras y estrategias dentro del sistema político que delinean la práctica de ciudadanía. Aunque se reconoce que el Estado es una arena donde se dan múltiples procesos de negociación, para fines del artículo lo tomamos como una unidad aparte, "un cuerpo de instituciones que están centralmente organizadas alrededor de la intencionalidad de control con un determinado aparato de ejecución a su mando o como soporte" (Yuval-Davies, 1997a: 42).

Arabia Saudita representa un Estado construido sobre un sistema neo-patriarcal, acompañado por arreglos corporativos de poder, donde la familia al-Saud es el principal arquitecto de la sociedad, al conformar un sistema político monárquico autoritario. De este modo, la ciudadanía saudita se encuentra caracterizada por la existencia de un "contrato de parentesco" organizado en torno a la noción de que todos los ciudadanos pertenecen a la familia antes que al Estado, la familia dentro de este contexto reclama las principales lealtades de los ciudadanos (Joseph, 2005: 149-150). Hacia el interior de cada familia predominan las bases patriarcales donde las mujeres y los menores quedan excluidos de las jerarquías de autoridad y de toma de decisión.

La modernización del Estado trajo como consecuencia que los príncipes de la familia al-Saud sean considerados como los patriarcas y protectores de la gran familia (sociedad saudita), siguiendo los principios de las relaciones masculinas contenidas en un sistema neo-patriarcal (Sharabi, 1980: 6-9). De acuerdo con 
Ayubi, los arreglos corporativos operan con base en las comunidades que refuerzan el poder del Estado, donde el grupo dominante en términos políticos y sociales, la familia al-Saud, sostiene una política de incorporación diferenciada hacia los distintos grupos sociales para alcanzar sus propios objetivos (Ayubi, 1999: 192-193). En este sentido es el Estado quien distribuye los recursos materiales y simbólicos a los diferentes grupos de acuerdo con sus intereses.

El principal círculo donde se distribuye la riqueza es la familia real. Las concesiones de negocios y los privilegios individuales a través de prácticas clientelares y de relaciones de familia son una expresión del acuerdo corporativista. La política de inclusión y exclusión del Estado y la alta dependencia de los diferentes grupos sociales dificulta la autonomía y solidaridad entre los mismos. Bajo este patrón de relaciones las mujeres son consideradas como indispensables para la reproducción y mantenimiento del sistema.

Como consecuencia de este sistema, mujeres y hombres en su mayoría se encuentran excluidos del proceso de toma de decisiones, a excepción de los príncipes con mayor rango dentro de la familia al-Saud. A nivel de la administración del gobierno, solamente por designación es que participan en puestos ministeriales y como miembros de los consejos consultivos (sin poder decisorio) hombres no pertenecientes a la familia real. Este proceso de inclusión en estos puestos se ha dado a través del tiempo, abriéndose un poco más a partir de la década de los noventa como resultado de la aparición de grupos disidentes.

El sistema de gobierno y el Estado se encuentran vinculados fuertemente a la religión, ya que su fundación ha estado ligada al cumplimiento de la misma, al igual que su legitimidad. La sumisión al Islam y la obediencia al emir fueron las bases sobre las cuales se construyó el nuevo estado en 1932. El pacto entre el Emir Muhammad Ibn Saud y el líder religioso Muhammad ibn Abdul Wahhab ${ }^{4}$ en 1745 simbolizó la alianza entre el poder político y el religioso que siglos más tarde daría lugar a la formación del reino de Arabia Saudita. Como consecuencia, en la actualidad los ulema oficiales (estudiosos de la religión encargados de las funciones religiosas por el Estado) junto con el Estado son las voces principales en la interpretación del pasado y en la construcción de la ciudadanía, noción que se ha ido transformando. De este modo, el ejercicio de ciudadanía se encuentra mediado por los usos locales y sujeto a la sanción religiosa, donde los ulema oficiales junto con las autoridades estatales, definen las prácticas del ciudadano y del buen musulmán.

El énfasis en el papel del Islam como parámetro de las prácticas tanto del Estado como del ciudadano se refleja en el sistema básico de regulaciones (Saudi Arabia-Constitution, 2008). ${ }^{5}$ El primer artículo sostiene que el Corán y la tradición del profeta es la constitución del país, y que el gobierno deriva su poder de las mismas fuentes (articulo 7); dadas las fuentes de poder y el papel central de la religión, los ciudadanos deben guardar alianza con el Rey (artículo 6). Este sistema otorga un papel central a la familia como la base de la sociedad y en la cual los individuos crecen en el conocimiento de Dios, siendo la principal función del Estado proveer los medios para fortalecer esta unidad familiar, y por ende la de la nación (capítulo 3). El Estado en estas regulaciones se presenta como el garante y proveedor del trabajo, de la educación, de la salud, de la protección de los derechos y seguridad de los ciudadanos.

En cuanto a los lineamientos para definir la ciudadanía se requiere que un ciudadano saudita sea aquel que haya estado viviendo en tierras sauditas desde 1914, ya sea registrado o no como ciudadano otomano, ${ }^{6}$ al igual que el individuo nacido de padre o madre saudita. Sin embargo, el Estado refuerza el sentido patrilineal de las relaciones de pertenencia al solicitar más condiciones para el otorgamiento de la ciudadanía al hombre extranjero casado con una nacional saudita que a una mujer extranjera casada con un saudita. Lo mismo pasa con la descendencia de estas parejas; en el primer caso los hijos no adquieren automáticamente la 
ciudadanía, pues están condicionados a un proceso de naturalización, mientras que en el segundo caso los hijos adquieren automáticamente la ciudadanía, (Ministerio del Interior, 2006).

Como señala Altorki, los elementos de ciudadanía presentes en el reino están altamente atenuados y el comportamiento de la ciudadanía parece más un comportamiento de súbditos que de personas con derechos y obligaciones (Altorki, 2000: 224). Debido a los arreglos del sistema político y social, las mujeres enfrentan múltiples formas de exclusión, como grupo que no tiene acceso a los procesos de toma de decisión, y en su misma condición de mujeres dependiendo de la clase social, status familiar y pertenencia a grupos étnicos y/o religiosos. ${ }^{7}$ Estos arreglos políticos le confieren al sistema de gobierno saudita un poder enorme para proveer acceso material y simbólico a los grupos de su preferencia. La riqueza económica generada por el petróleo y la interpretación del Islam son las bases del poder político y son utilizadas por las autoridades para influir en la percepción de las diferencias entre hombres y mujeres. Aunque hay que destacar que las mujeres en ningún momento han sido solamente receptáculos de estas políticas, pues a través del tiempo y su localización en la sociedad no siempre son seguidas, sino también transformadas y apropiadas por las mujeres.

\section{La recreación de la ciudadanía}

Teniendo en cuenta las características del sistema político y social de Arabia Saudita, podemos notar cómo las regulaciones sobre la mujer son parte de la relación de cooperación entre los ulema oficiales y el gobierno. Si se sigue el principio patriarcal, las mujeres están sujetas a políticas que las hacen responsables del mantenimiento del honor de la familia y las tradiciones, sujetas a la aprobación del hombre de la casa y del Estado.

En términos religiosos la asignación de roles queda reflejada en la opinión dada por el ya fallecido Mufti ibn
Baz, ${ }^{8}$ el rey es el guardián de sus súbditos, el hombre es el guardián de la familia y la mujer es la guardiana del hogar de su esposo, siendo responsable de su casa y de educar a la familia en el conocimiento de Dios (Ibn Tiar 1992: 348). El gobierno también enfatiza el lugar de la mujer al considerar que ésta tiene una función especial "al ser la fundación societal al cuidado de educar a los niños y prepararlos para servir el país y educarlos en varios campos" (Ministerio de Información, 2004).

Las mujeres de acuerdo a la interpretación de los ulema, pertenecen principalmente al ámbito privado y las funciones que realizan dentro de la esfera doméstica y fuera de ella son de acuerdo a su naturaleza femenina. La interpretación y aplicación de la ley religiosa, apoyada en políticas públicas, permite al gobierno fluctuar en interpretaciones rígidas o flexibles, de acuerdo con sus necesidades al recaer principalmente en la mujer (Doumato, 1995: 145). Socialmente esta diferencia queda legitimada por el valor asignado a la cuestión de honor, por lo que la reclusión de las mujeres es la práctica impuesta para reforzar esa virtud. Bajo esta perspectiva, los hombres son considerados los fuertes, los protectores, los proveedores; mientras las mujeres son consideradas las débiles, desprotegidas, las dependientes (Altorki, 1987: 67). Para la sociedad saudita el código de honor (ird) es un valor muy importante, pues el honor de la familia descansa en el comportamiento de la mujer, y evitar exponerse a otros hombres que no sean de su familia es considerado un buen comportamiento. Las políticas del uso del velo y la cubierta del cuerpo con una túnica negra (abaya) tienen como objetivo evitar esta exposición y proteger a la mujer. Por ello es que existe un gran número de opiniones religiosas acerca de la vestimenta y comportamiento de la mujer. ${ }^{9}$

Esta asignación de roles está acompañada por la división del espacio público y privado, masculino y femenino respectivamente, y se establece mediante la cooperación entre el Estado y los ulema oficiales. De acuerdo al mufti Ibn Baz es imperativo evitar las mezcla de hombres y mujeres, ya que conduce al mal y la depravación (Al Mus- 
nad, 1996)..$^{10}$ Las áreas del espacio público se encuentran divididas entre los espacios exclusivos para los hombres y los espacios para las mujeres y la familia."

La segregación de espacios también permea todas las áreas de la vida de las mujeres y limita sus aspiraciones. Las elecciones de la mujeres acerca de su educación tienen que ver con la autorización de su guardián legal (wali al amr) y deben elegir entre las carreras que son consideradas apropiadas a su condición de mujeres, tales como enfermería, medicina, educación y carreras de tipo administrativo. ${ }^{12}$ En el año académico 1977-1978 el número de mujeres que asistían a la universidad fue de 5,658, representando 18\% del número total de estudiantes universitarios (Ministerio de Educación Superior, 1980). Para el año de 1989-1990 el porcentaje de mujeres se incrementó al llegar a 53,030 estudiantes mujeres, lo cual representó 47\% del total de alumnos inscritos en ese nivel. En años recientes el número de mujeres con estudios universitarios continuó aumentando. En las áreas de trabajo la situación es similar, pues requiere la autorización de su guardián y solamente está autorizada a trabajar en lugares donde se respete la segregación; generalmente los trabajos son en el sector público y un porcentaje bajo en el sector privado. Aunque recientemente el gobierno en sus planes de desarrollo ha animado la participación de las mujeres en la creación de empresas, éstas tienen que respetar los códigos de los espacios segregados y por lo general han tenido que contar con la representación de un hombre en la obtención del registro y los trámites. En la actualidad las mujeres dueñas de empresas representan $3.4 \%$ y se encuentran dedicadas a la construcción, manufactura, decoración, educación e industrias relacionadas con productos para las mujer y familia, (Ministerio de Información, 2004: 92).

\section{El enfrentamiento con el Estado}

Como señala Sundran, en la relación entre el Estado y "las mujeres" ambos se definen, pues estos actores se constituyen en el momento de su interacción. El primero como objeto de la movilización con un interés social creado y el grupo como entidad específica al cual el Estado dirige políticas y las identifica como grupo al momento de la movilización. Esta relación se caracteriza por ser asimétrica, pues frecuentemente es el Estado quien establece los parámetros y las mujeres son más bien reactivas. Sin embargo las mujeres en situaciones específicas lo hacen reaccionar, adquiriendo visibilidad (Sundran, 2003: 24-25). La segunda guerra del Golfo fue el contexto para el surgimiento de oposición organizada y pública. La dependencia con Estados Unidos y con las fuerzas occidentales en general para defender al reino, fue objeto de crítica en el mundo árabe y hacia el interior del reino. A nivel social, la política de reforzar los valores islámicos y el papel de los ulema para legitimar la entrada de tropas extranjeras al territorio saudita abrió espacios para la resistencia y reinterpretación del Islam con la finalidad de enunciar una crítica más amplia al sistema. ${ }^{13}$ Para nuestros propósitos este contexto particular es importante, pues por primera vez un grupo de mujeres sauditas se manifestó públicamente en Riyad por el derecho a conducir en Arabia Saudita. Cómo las mujeres tomaron ventajas de este contexto político y cómo el gobierno y la sociedad reaccionaron es el objetivo de esta sección.

La situación de relativa apertura durante este contexto, y la presencia de la prensa internacional fueron usadas como una oportunidad para las 47 mujeres que se manifestaron públicamente. El rey Fahd había solicitado la aceptación de mujeres voluntarias para apoyar las tareas de enfermería y las actividades humanitarias; el gobierno organizó comités para coordinar el registro y entrenamiento de las mujeres. En todas las provincias las mujeres inmediatamente respondieron al llamado al sentirse tomadas en cuenta en la defensa del país (Arab News, 13 de septiembre de 1990). Había tal sentido de patriotismo en el país que tanto hombres como mujeres se alentaban unas a los otros para poder participar en los diferentes frentes propuestos por el gobierno (Saudi Gazette, 17 de septiembre 1990). 
Un grupo de mujeres de Riyad, de clase media, educadas y con experiencia de vida en el extranjero consideraron que ese era el momento para solicitar el derecho a conducir. Al principio las reuniones se efectuaban en un pequeño círculo de amigas, quienes acostumbraban a reunirse en sus propias casas con la finalidad de intercambiar opiniones acerca de la vida diaria y de sus actividades profesionales. Con el paso del tiempo, estas reuniones empezaron a atraer más mujeres hasta llegar a cuarenta.

Este grupo de mujeres consideró que el discurso del gobierno señalaba la necesidad de un papel másfuerte de la mujery ante sus limitaciones para desplazarse, pues los hombres se habían ido a la frontera con Irak y Kuwait, al mismo tiempo muchos de los trabajadores inmigrantes habían salido del país; consideraron que una manifestación podía crear presión en el gobierno y otorgarles el permiso para conducir. ${ }^{14}$ En este sentido es que se puede explicar la postura de las mujeres en su carta al gobernador de Riyad, antes de hacer la manifestación.

Este grupo retomó el discurso del gobierno de llamar a las mujeres a defender la nación, lo que se expresó en su consideración de retribución a un país que les había dado bienestar y que en ese momento de grandes retos, se requería la contribución de todos en unidad, hombres, mujeres. Las mujeres justifican su solicitud no solamente en términos de su compromiso con la nación, pero también en términos sociales y religiosos donde declaran que el depender de los choferes (hombres inmigrantes), ocasionaba problemas en el hogar y que no era aceptable que las mujeres estuvieran a merced de un extraño, además ante la situación de crisis las mujeres podían ayudar al hombre (Petición de las Mujeres Sauditas al Emir de Riyad).

Estas mujeres se organizaron y acordaron usar la tarha (velo que cubre el cabello) y la abaya, todas contaban con licencia de conducir internacional. Su manifestación consistió en manejar por una calle principal de la ciudad por un periodo de 15 minutos; en su recorrido fueron arrestadas. Una de ellas contactó a dos medios internacionales, CNN y BBC para pedirles que cubrieran la noticia. Como una de las participantes explica:

Estoy contenta que ella hiciera eso, nuestra voz alcanzó el mundo exterior, de otro modo nosotros hubiéramos estado en otra situación (entrevista personal, Riyad, 27 de enero 2007).

La sociedad reaccionó en contra de la manifestación, al convertir este hecho en un escape de la situación de incertidumbre y temor que enfrentaba el reino ante las posibilidades de un conflicto con Irak. Las mujeres fueron detenidas por la policía durante toda la noche, eventualmente fueron puestas en libertad, sus pasaportes junto con el de sus esposos fueron confiscados, las mujeres fueron forzadas a abandonar sus trabajos y sus esposos fueron obligados a firmar una carta en donde estipulaban que sus mujeres no iban a conducir. De acuerdo con algunas de las participantes ellas enfrentaron el desacuerdo de sus propias familias. Una de las participantes relata: "mi padre no quería que yo o mi propia familia tuviéramos contacto. En mi trabajo hasta estos días mis superiores y colegas no quieren saber mucho acerca de mí. Tengo que cubrir mi cara, usualmente no me la cubro, pero al entrar en mi edificio de trabajo lo hago con la finalidad de no afectar a mis colegas" (entrevista personal, Riyad, 9 de junio 1999).

En el caso de seis maestras de la universidad Rey Saud, a pesar de que tenían el apoyo de algunos grupos de estudiantes y colegas en los primeros días después de la manifestación, la oposición en contra de ellas surgió. En ese tiempo los departamentos Islámicos estaban creciendo, y desde ahí se organizaban juntas y manifestaciones denunciando la conducta de las maestras y su mala influencia para los estudiantes. Las maestras fueron forzadas a retirarse del trabajo y las autoridades firmaron una carta donde se deslindaban de lo sucedido.

Inmediatamente después del incidente, propaganda revelando el nombre de la mujer participante y su 
esposo circuló en todo el reino. En los sermones de los viernes estas mujeres eran consideradas como lo peor de la sociedad, tanto por los ulema oficiales como los no oficiales. De acuerdo con uno de los esposos de las participantes él recibióllamadas insultantes y fue objeto de bromas e ironías en su lugar de trabajo, respecto a su capacidad para controlar a su esposa (entrevista personal, Riyad, 4 de marzo 2007).

Los ulema oficiales inmediatamente elaboraron una opinión religiosa ( fatwa) declarando que el hecho que la mujer condujera era en contra del Islam. Esta opinión argumentaba que si la mujer conduce podría ocasionar depravación y enfatizaba el confinamiento de la mujer a la esfera privada.

...Las mujeres manejando conducen a muchos males y consecuencias negativas, incluyendo entre éstos la mezcla con hombres sin estar ella alerta. También conduce a pecados por ello tal acción está prohibida.... Las leyes que purifican prohíben todas las causas que conducen a la depravación... (Ibn Baz, citado por al Musnad 1996: 310-311).

Por su parte el gobierno a través del ministro del interior, príncipe Naif, anunció la prohibición oficial a las mujeres, tanto a las sauditas como a las extranjeras residentes, basado en la fatwa del Consejo Superior de los Ulema, añadiendo que habría un castigo adecuado para los violadores de esta regulación, y enunciando que esta prohibición tenía como finalidad proteger la santidad y prevenir la degradación de las mujeres (Saudi Gazette, 14 de noviembre 1990). Más tarde el príncipe también en un discurso público señaló que las mujeres manifestantes fueron educadas en el extranjero y recibieron educación no religiosa, previniendo a los jóvenes de seguir "ideologías destructivas y cultura sospechosa importada" (Saudi Gazette, 16 de noviembre 1990).

Para los diferentes grupos en oposición, la manifestación de las mujeres era una prueba más de que no estaban honrando su compromiso con el Islam. Dos prominentes líderes de la oposición, Sheik Safar al Hawali y Sheik Salman al Auda denunciaron el acto de las mujeres como una táctica para corromper a la familia y sociedad en el reino (Movimiento para la Reforma Islámica en Arabia, 1999). Tanto el gobierno como los ulema oficiales actuaron de manera conjunta, al condenar la manifestación y prohibir a las mujeres conducir. El gobierno explotó el hecho y distrajo la atención pública de la polémica levantada por la presencia de tropas extranjeras. Los ulema oficiales con su postura en contra de la manifestación reafirmaron su autoridad sobre los elementos simbólicos y culturales de la sociedad, especialmente frente a la oposición.

De este modo la manifestación pública y abierta de las mujeres no encontró una respuesta positiva, al contrario, el Estado dejó caer el peso de la ley y la religión en las sanciones otorgadas a estas mujeres, y al hacerlo dentro del contexto de la II guerra del Golfo, la mujer se convirtió en el símbolo de su cumplimiento con la religión y con la defensa de la nación.

\section{Hacia la construcción de una ciudadanía activa}

El solo hecho de que estas mujeres se organicen y presenten nuevos roles a la sociedad nos habla de la construcción de una ciudadanía activa retomando la tipología propuesta de Turner. La ciudadanía activa implica una actividad por parte de los ciudadanos de participar en la defensa de la democracia (Turner, 2000: 43-45), "a nivel individual se expresa como participación; a nivel social como responsabilidad, solidaridad y cooperación en la esfera público-social; a nivel político como exigencias de fiscalización y rendición de cuentas de lo público-privado" (Vargas, 1997:58-59). Sin embargo esta construcción de ciudadanía por parte de las mujeres en Arabia Saudita está en sus inicios, en comparación con otras naciones árabes musulmanas donde hay una historia importante de movimientos de mujeres como Egipto, Turquía o Irán entre otros. Estos inicios pasan por la negociación en la esfera privada y la construcción 
de espacios donde se articulan rutas alternativas a las regulaciones sociales y políticas, y donde las prácticas de resistencia y discurso son socializadas sin confrontar al Estado (Scott, 2000: 147-148). En contraste, las acciones de este grupo de mujeres en los inicios del nuevo milenio son consideradas como prácticas de resistencia abierta, ${ }^{15}$ la cual hace referencia a la conducta que es visible einmediatamente reconocida tanto por sus objetivos y observadores como resistencia, "además sus actores la reconocen como tal" (Hollander y Einwohner, 2004: 545) $\cdot{ }^{16}$ En esta sección analizaremos las características del perfil de las mujeres activistas para posteriormente comprender las formas de articulación de sus demandas, en los posteriores incisos.

En términos generales podemos enunciar que las mujeres pertenecen a la clase media con estudios de postgrado y las podemos ubicar en tres generaciones. La primera generación creció en la década de los sesenta y setenta, cuando el Estado estaba en pleno proceso de consolidación y existía una mayor flexibilidad. Por lo general estas mujeres fueron apoyadas tanto por el padre o la madre, en casos en los cuales el padre había fallecido, para seguir con su educación universitaria. En la mayoría de los casos estas mujeres al no tener educación disponible en el reino, fueron a países de la región a continuar con sus estudios universitarios, algunas a países occidentales. Sus padres pertenecían a una categoría intermedia-alta en el gobierno, y el hecho que permitieran a sus hijas salir del país y continuar con su educación era algo que iba en contra de las prácticas sociales, o aún más en caso de fallecimiento del padre y la misma madre -aunque no era educada- motivó y luchó por que sus hijas continuaran estudiando. Salvo en dos casos, la forma en que se negoció la continuación en la educación y la salida del país fue a través del casamiento.

Los años en los que transcurrieron los estudios de estas mujeres fueron de una intensa actividad política y cultural, destacándose los eventos relacionados con el problema árabe-israelí, el movimiento feminista en occidente, la intervención de Estados Unidos en
Vietnam; lo que ocasionó la toma de conciencia acerca de la importancia de la solidaridad y la resolución de problemas dentro de su propia comunidad y a nivel regional.

El mayor número de mujeres participantes se concentra en esta segunda generación. Este grupo de mujeres fue el que experimentó el cambio de políticas hacia la mujer y hacia la defensa del Islam en el Reino, después de la toma de la Meca en 1979 por un grupo de disidentes y la llegada al poder del ajatolla Jomeini. En cambio la tercera generación que accedió a la educación universitaria en los noventa, fueron las mujeres que crecieron en un sistema rígido con respecto a la aplicación de políticas hacia la mujer y donde empiezan a aparecer los síntomas de crisis económicas ocasionadas por los cambios en el precio del petróleo y la factura de los gastos del conflicto ocurrido a partir de la invasión de Irak a Kuwait.

El segundo grupo de mujeres pudo tener acceso a la educación universitaria proporcionada por el Estado durante la década de los ochenta. Como señala Hala:

...antes del levantamiento de Yuhaiman y de la llegada del ayatolla, nosotros teníamos educación física, teníamos festivales con música al término del ciclo escolar, después de los sucesos no se nos permitieron ni la educación física, ni la música en nuestros festivales (Entrevista Personal, Riyad, 10 de febrero 2007).

De acuerdo a Faisa:

...nuestros planes de estudio eran como los de cualquier otro país en la región, incluyendo a nuestro cuerpo de profesores que provenían de diferentes partes del mundo árabe. Después en los ochenta la educación fue secuestrada por religiosos, los planes de estudio y las regulaciones de la educación quedo en sus manos (Entrevista personal, Riyad, 27 de diciembre 2006). 
En términos de la vestimenta, Faisa recuerda con enojo cuando recibió la amonestación a la entrada de la primaria por no usar la abaya, "nadie debería tener el derecho de castigar o de gritar a nadie". Faisa señala que antes la gente podía usar una cubierta hasta debajo de las caderas, pero durante los ochenta se hizo obligatorio que las mujeres se cubrieran de negro.

Cuando se les preguntó a las mujeres activistas cómo es que surgió su interés y percepción acerca del rol de la mujer surgen tres variables, la primera es por el modelo de familia que imperaba en su casa, impuesto por el mismo padre, donde había igualdad; el segundo es a través de la literatura regional y occidental; y por último las experiencias de las mujeres en su entorno familiar, la pérdida del padre, ya sea por muerte o por divorcio y el papel de la madre para sacar adelante a la familia.

Algunas de estas mujeres activistas desde los ochenta han creado roles alternativos para las mujeres, la creación de talleres de computación en los primeros años de la década de los ochenta, o han construido negocios relacionados con la salud, educación, vestimenta, a pesar de las limitaciones. Otras han incursionado en campos tradicionalmente asociados con hombres, como la construcción, la poesía y la escritura de columnas o pequeñas historias en los periódicos, desde antes de los ochenta. ${ }^{17}$ Algunas de estas mujeres han sido encarceladas por plasmar críticas en sus escritos en contra del gobierno, o por ser sospechosas de tener nexos con grupos oposicionistas, y a otras se les ha limitado y/o obstaculizado el ejercicio profesional y de sus estudios de postgrado, durante la década de los ochenta y noventa.

Después de la manifestación pública de las mujeres y a pesar de la condena social y del Estado, las mujeres en general no han dejado de intentar prácticas y espacios para rebasar las limitaciones, incluso se puede decir que reanimó la práctica de crear y asegurar sus propios espacios. En la historia del reino, una práctica común para las mujeres de estratos superiores y medios de la sociedad, ha sido la formación y participación en obras de caridad. Estas organizaciones caritativas han contado siempre con la participación de miembros de la familia real. Como señala Fakro, en las sociedades del Golfo estos pueden ser los canales mediante los cuales se puede realizar un cambio (Fakro, Munira, 1996: 260).

Durante los noventa y especialmente hacia la segunda mitad, los espacios de mujeres crecieron. Las mujeres en estos espacios construyeron redes sociales que les permitieron sentar las bases de lo que eventualmente sería el núcleo mediante el cual se podría abrir la discusión y flexibilización a las políticas gubernamentales hacia la mujer. Aunque no todos tuvieron una connotación de resistencia abierta, constituyen espacios de socialización de discursos que cuestionan el orden o buscan participar en el servicio a la comunidad en su calidad de proveedoras del cuidado. Estos espacios fueron de tipo religioso, salones literarios y grupos centrados en alguna actividad en particular como negocios o formación de asociaciones para luchar contra problemas de la comunidad. Estos encuentros hasta el año 2007 se hacían sin permiso oficial, así que las mujeres participaban voluntariamente, ofreciendo sus casas y recursos para la realización de estas reuniones y actividades.

\section{La politización del papel de la mujer}

La sociedad saudita a principios del nuevo milenio aparecía dividida entre la unidad y la diversidad. A los procesos de la globalización fueron añadidos el descubrimiento de la participación de nacionales sauditas en los ataques terroristas del 11 de septiembre, al mismo tiempo que las actividades terroristas también estaban en casa. Ni el gobierno, ni la sociedad podían ignorar esta realidad, la cual se agudizaba con los problemas regionales: la ofensiva estadounidense a Afganistán e Irak. Estos peligros produjeron en algunos momentos y temas la unidad, pero también la división entre diferentes grupos de la sociedad. En el 2003 se formularon varias peticiones públicamente al gobierno y un grupo de mujeres por primera vez las firmó y formuló una petición propia. 
Nuevamente como en la anterior coyuntura nos enfrentamos a un momento de amenaza para el país; las mujeres del grupo de Riyadh, especialmente, participaron en el debate acerca de la defensa de la nación, al reclamar un lugar y su completa participación como ciudadanas en tres momentos y estrategias entrelazadas. Primero en la presentación de demandas a partir de un grupo de mujeres de Riyad, segundo al trabajar con el gobierno participando en el primer diálogo nacional acerca del papel de la mujer, y por último, la participación de las mujeres en las elecciones municipales y en las elecciones de las cámaras de comercio en Jedda y Damam. Para retomar las líneas de debate es necesario explicar y analizar su contenido entre los diferentes grupos de la sociedad. Posteriormente se presentarán las estrategias de las mujeres para aprovechar esta coyuntura, así como también comprender por qué ahora si respondió efectivamente el gobierno ante las demandas de las mujeres.

El resurgimiento de una oposición Islamista muestra la incapacidad del gobierno por frenar las actividades de estos grupos. El aumento de sus actividades y en algunos casos su radicalización nos muestra su descontento con el gobierno de Arabia Saudita y el papel de occidente en la región, particularmente Estados Unidos (Galindo, 2007: 259-288). De manera general los diferentes grupos de oposición se pueden intensificar en tres grandes categorías: Los “liberales" profesionistas, intelectuales, personas con una trayectoria de disidencia desde años atrás. El segundo gran grupo es el de los islamistas, con una serie de divisiones hacia el interior (Lacroix, 2004: 4). Un grupo encabezado por los líderes oposicionistas de la década de los noventa y que ahora han sido cooptados por el gobierno, presenta una postura reformista. Otro grupo de islamistas es el identificado con la oposición de los noventa, pero que mantiene una postura independiente respecto a los primeros. Por último el grupo radical está conformado por personas que están comprometidas con el yihad (guerra santa externa) para derrocar a la familia real y su gobierno, y eliminar la presencia occidental del territorio saudita. Este último grupo tiene lazos con Al Qaeda y ha sido responsable de los ataques terroristas en el reino durante el 2003.

En el 2003 fueron presentadas al príncipe heredero Abdulla tres peticiones, siendo la primera vez en la que algunas mujeres participaron en la firma de dos de ellas. La primera petición fue formulada por el grupo de liberales e Islamistas reformistas. La segunda petición fue presentada por la minoría chiíta, entre los 450 que firmaron la petición, 24 fueron mujeres. La tercera petición fue presentada como una reacción a los ataques terroristas de mayo de ese año y fue firmada por 300 gentes, incluyendo a 51 mujeres.

El común denominador de estas tres peticiones es el llamado a una sociedad plural y participativa. La incorporación del respeto a la diversidad, enfatiza la necesidad de cambiar el carácter exclusivo de la entidad wahabita, y se señala la necesidad del gobierno de respetar y proteger los derechos de todos sus ciudadanos. La solicitud de abrir el sistema para la participación en los procesos de toma de decisiones, así como el otorgamiento de derechos para el establecimiento de instituciones civiles fue considerada como el antídoto para luchar contra el extremismo y defender a la nación.

De las tres peticiones solamente la primera enunció la participación de la mujer como importante, siguiendo los parámetros de la ley islámica (sharia) y dentro del rubro protección y peligros a la nación. Esta petición fue presentada de manera conjunta por los liberales, los islamistas reformistas y los principales líderes de la comunidad chiíta. Por ello, cuando los liberales presentan una petición por separado, teniendo como finalidad conseguir más apoyo, recurren a la inclusión de las mujeres en el respaldo a su petición. Como uno de los participantes en la elaboración de la primera y tercera petición señaló: "en la primera necesitábamos formar un frente común con los islamistas, pero en la segunda cuando solicitamos a las mujeres su firma, algunas se mostraron renuentes debido a los costos que podrían enfrentar, y otras mujeres querían una ampliación de 
las demandas sobre el papel de las mujeres" (Jaled, entrevista personal, Damam, 19 de febrero 2007). Las mujeres que dieron su firma a esta última petición lo hicieron por el simple hecho de que se enunciaba el papel de la mujer.

Ante esto tenemos que en este debate y en la estrategia de algunos grupos como el de los liberales, lo principal es la liberalización del sistema para permitir a los ciudadanos participar, aunque este tipo de participación sea más bien masculina. La lucha de las mujeres entonces queda relegada a un segundo plano, como si el ejercicio de la obtención de las libertades fuese solamente prerrogativa de los hombres. Dentro de este debate y considerando el contexto de la sociedad saudita, el hecho de hablar a favor de la mujer es considerado por muchos como si fuera algo no islámico y más bien secular de acuerdo a un opositor saudita en el extranjero (entrevista personal, 22 de julio 2004).

Ante esta falta de voz y de consenso, las mujeres del grupo de Riyad decidieron presentar una petición propia por primera vez en la historia del Reino. La formulación de esta petición fue objeto de numerosas reuniones para acordar el contenido y además para retomar las opiniones y demandas de otros grupos de mujeres. La labor del grupo se extendió a lo largo y ancho del país, utilizando Internet como principal medio de comunicación. Finalmente la petición fue firmada por más de 300 mujeres y no se hizo pública, sino que fue entregada a la Princesa Adela, hija del rey Abdulla, a través de un pequeño comité de mujeres. La petición se publicó posteriormente al final del año.

Las demandas contenidas en esta petición incluyeron los derechos respecto a su propia agencia como sujetos: el reclamo de derechos de tipo social, político, legal y económico. Con respecto al primero, las mujeres en su primera demanda solicitaron la desaparición de la figura del guardián (wali al-amr), enfatizando con ello los derechos de la mujer para decidir por ella misma y gozar de su derecho a casarse, a disfrutar sus posesiones, el derecho a obtener la custodia legal, a estudiar y a expandir las área de educación para las mujeres. Las mujeres dentro de la esfera política reclamaron su derecho a participar en los procesos de toma de decisiones tanto en organismo estatales como privados, solicitando regulación al respecto para poder implementar este derecho y dar lugar al desempeño de la mujer en puestos directivos. Asimismo demandaron el establecimiento de cuotas para la participación de mujeres y otros grupos representativos de la sociedad en la Asamblea Consultiva. La conformación de un Consejo Nacional para la Mujer, lo consideraron importante, pues este organismo vigilaría la implementación de las regulaciones por parte del Estado. En materia legal, reclamaron el goce de igualdad de derechos entre varones y mujeres sauditas, pidieron la codificación del derecho de la familia y el desarrollo de cortes de lo familiar, así como también la aceptación de otras escuelas de jurisprudencia y sus opiniones religiosas acerca del rol de la mujer para contribuir a su mejora. En el caso de los derechos económicos las mujeres pidieron la provisión de seguridad social para todas las desempleadas (Al Fassi, 2003).

Aunque no recibieron una respuesta oficial directamente, las mujeres lograron que la discusión se orientara hacia su papel en la sociedad. El Rey anunció la celebración de un diálogo nacional sobre el papel de las mujeres. Las mujeres participantes en la petición trataron de aparecer en periódicos y en programas de televisión para hablar sobre los derechos de las mujeres, e influenciar con ello la agenda del encuentro. En este sentido es importante destacar que una gran parte de las mujeres invitadas a participar pertenecía al grupo de mujeres demandantes. El mismo grupo ayudó a estas mujeres a prepararse para el encuentro, especialmente en religión, pues sabían que iban a estar presentes elementos conservadores y religiosos en el diálogo.

Aunque para algunos sauditas los resultados del tercer diálogo no fueron positivos, pues imperó la visión conservadora y tradicional de la sociedad y no obstante el prolongado silencio sobre el tema de las mujeres en Arabia Saudita; el simple hecho de que este diálogo se 
haya efectuado es un avance. Además resulta alentador que entre las recomendaciones del encuentro estaba el establecimiento de una organización encargada de resolver los problemas de la familia y la mujer, al igual que un comité de expertos en sharia para remover las tradiciones y costumbres de los derechos de las mujeres. Esto último muestra un avance respecto a la década anterior donde hay un reconocimiento sobre el papel de las costumbres y de lo social en la configuración del papel de la mujer, más que la propia religión. Lo anterior permite reflexionar sobre los márgenes de maniobra tanto del gobierno, como de las mismas mujeres. Si en la década anterior el Príncipe Naif había declarado que se prohibía a las mujeres conducir de acuerdo con el Islam y sus tradiciones, ahora este permiso lo considera sujeto a la sociedad.

\section{La participación en las elecciones}

Otra área donde las mujeres intentaron participar fue en las elecciones para ocupar la mitad de los asientos en las municipalidades ${ }^{18}$, las cuales se realizaron por primera vez en la historia del reino en el 2004. Dado que las regulaciones relativas a las elecciones no especificaban el sexo de los candidatos y sólo mencionaban que el candidato debía tener una edad mínima de 21 años y no pertenecer a las fuerzas armadas (BBC Middle East News, 10 de agosto 2004), algunas mujeres sauditas aprovecharon esta oportunidad. Antes que el encargado de las elecciones presentara alguna resolución al respecto, algunas mujeres en Riyad empezaron a presentarse como candidatas y otras escribieron artículos a favor de la participación de la mujeres en las elecciones, de tal modo esto influyó para que otras candidatas de otras regiones se presentaran.

Las mujeres que lanzaron sus candidaturas acordaron que sus mayores motivaciones eran su deseo por mostrar a la sociedad que las mujeres son capaces de organizarse y de sobrepasar las limitaciones haciéndose visibles, así también mostraron un interés por la pro- blemática de sus comunidades. De acuerdo con una de las participantes, "es mi derecho a participar, no es la opinión pública quien decide si participo, debería ser el gobierno, pero sigue siendo mi derecho el decidirme a participar". Las mujeres candidatas fueron apoyadas por grupos de mujeres de su propia ciudad. Cada una se concentró en el trabajo que podría aportar a la problemática municipal de acuerdo con sus habilidades y preparación.

El grupo de Riyadh otra vez mostró actividad y solidaridad con las mujeres al apoyarlas en la organización y presentación de sus plataformas políticas. El contenido de las plataformas incluyó problemáticas de la comunidad que tomaran en cuenta a la mujer, a los jóvenes y a los adultos mayores. Cuestiones como el cuidado del medio ambiente y regulaciones sobre la construcción fueron los puntos importantes de sus agendas políticas.

Además como antes lo habían hecho, la apropiación de espacios de comunicación tanto a nivel nacional como internacional, jugó a su favor. La participación de las mujeres en los programas de televisión apoyando la participación de la mujer, así como también el mostrar públicamente que existen precedentes dentro de la religión, desde tiempos del profeta, donde las mujeres han participado en los asuntos públicos; contribuyó nuevamente a hacer visible el papel de la mujer. El hecho de que en la sociedad se discutiera sobre la participación de la mujer y se entrevistara a las propias candidatas constituía una táctica importante. Por otro lado, las mujeres no desistieron de presionar a la parte oficial, el comité encargado de las elecciones, para que se pronunciara a favor de la participación de la mujer. No obstante, el Ministro del Interior en octubre del 2004 emitió la resolución en contra de la participación argumentando que las autoridades carecían de los medios técnicos para adecuar las votaciones para las mujeres. Para una de las organizadoras, el hecho que el pronunciamiento no haya sido en torno a una justificación religiosa o social fue una victoria importante para las mujeres. De acuerdo 
con una de las participantes, Nadia Bukhurji: "no estaba sorprendida, pero estaba decepcionada. Como mujeres esperamos cierta oposición a ser empoderadas, aunque en términos legales y religiosos estamos completamente dentro de nuestros derechos como ciudadanas" (BBC News, 4 de julio 2005). Para otras participantes "el hecho que la prohibición fue justificada en procedimientos y no en términos religiosos o sociales representó una victoria y una esperanza de que en las próximas elecciones las mujeres tendrán oportunidad de participar".

En el sector privado también un grupo de mujeres participó por primera vez en las elecciones para elegir a los miembros de las cámaras de comercio en Jedda y Damam. Lo anterior no es casual, pues en estas dos regiones las mujeres se han organizado y logrado una apertura de las cámaras para incluir una sección femenina, al tomar en cuenta la apertura de estas regiones al comercio y la "flexibilidad" en las normas y tradiciones religiosas. En el primer caso un grupo de 30 mujeres lograró el establecimiento del centro de mujeres de negocios en el 2001, bajo el auspicio de la Princesa Adela. Se nombró al centro Zayda Wahiya, en honor a una de las esposas del profeta, quien es considerada como una de las primeras mujeres en negocios. Con esto las mujeres evitaron la intromisión de la policía religiosa. En cambio en Damam no solamente las mujeres lograron el establecimiento de una sección de mujeres en la cámara de comercio, también otro grupo de mujeres de negocios formó una organización independiente, bajo el auspicio de la esposa del gobernador de la región, pero sin reconocimiento legal.

En las elecciones para los consejos de las cámaras, las mujeres en Jedda fueron invitadas por algunos de sus colegas masculinos, ganando dos asientos y otros dos lugares dados por el gobierno federal, por el ministro de comercio. En el caso de las mujeres de Damam participaron en las elecciones 4 candidatas, pese a que se les informó que podían participar con muy poco tiempo antes de que cerraran el registro. La presentación de sus candidaturas no fue fácil para algunas mujeres pues tuvieron la oposición de los miembros de sus familias extensas. No obstante, las mujeres de la organización independiente contaron con el apoyo de las redes de mujeres que se habían unido para apoyarlas en los puestos municipales. Aunque obtuvieron una gran cantidad de votos considerando que se presentaron de manera independiente, no fue suficiente para que ganaran las elecciones.

Las campañas en Damam, de acuerdo con dos de las candidatas, fueron sobre los temas de interés para el comercio: el tema de las pequeñas y medianas industrias, el buen manejo financiero en las empresas, la entrada de Arabia Saudita a la Organización Mundial del Comercio (OMC). Las mujeres contaron con el apoyo de jóvenes para invitar a la participación de sus eventos, donde siguieron las políticas de segregación. De acuerdo con una candidata "la carencia de dinero y la capacidad de cabildear de los hombres, especialmente a través de líneas tribales, fueron los mayores obstáculos que tuvieron" (entrevista personal, Damam, 19 de febrero 2007).

Las mujeres que presentaron sus candidaturas, tanto en las elecciones para las municipalidades como para las cámaras de comercio, comentaron cómo ellas se convirtieron, de acuerdo a algunos padres de familia en modelos de rol para sus hijas. Aunque también otras recibieron amenazas e insultos por parte de elementos conservadores de la sociedad. Algunas de ellas, pese a la negativa del gobierno o pese a perder las elecciones, están contentas con sus acciones, en términos del compromiso con su comunidad y con un mejor futuro para sus hijos. Cuando se les pregunto si volverían a intentar participar en las elecciones la respuesta fue positiva.

\section{Reflexiones}

En este artículo se ha tratado de descentrar el discurso de la ciudadanía basado en una genealogía occidental. Estudiar la ciudadanía en términos teóricos y prácticos en sociedades no occidentales nos provee de un marco de reflexión sobre los aspectos que inciden en su formulación. La ciudadanía occidental basada en 
la llegada de la modernidad se establece como pauta para tratar de encontrar las formas que adopta en otras sociedades. La realidad en los países en desarrollo, como hemos mencionado, es una realidad híbrida donde coexisten diferentes momentos anteriores y posteriores a la modernidad. Por lo tanto, la existencia de formas patriarcales, los lazos familiares a través de los hombres y la religión, son considerados elementos tradicionales y premodernos, en realidad se encuentran conviviendo con la modernidad, lo cual los hace parte y producto del mismo proceso en este tipo de sociedades.

Las condiciones políticas de Arabia Saudita en particular y de los demás Estados del Medio Oriente, donde sus sistemas son más bien autoritarios, contrarios a las prácticas y bases teóricas de la ciudadanía occidental basada en sistemas republicanos y democráticos, muestran que hay otras trayectorias de la ciudadanía. La progresión lineal de derechos de la ciudadanía apoyada en el modelo de Marshall se tendría que repensar para casos no pertenecientes a los países desarrollados. En el caso de Arabia Saudita, la manera como se ha "otorgado" estos derechos ha sido más bien una estrategia del gobierno para controlar la apertura del régimen, que una reivindicación de la defensa de los derechos por parte de los ciudadanos. Además aún así podemos notar que no todas las prerrogativas de la ciudadanía son reconocidas por el gobierno, ni practicadas por los ciudadanos y ciudadanas.

Los análisis occidentales acerca de las mujeres del tercer mundo como oprimidas dentro de un contexto de falta de desarrollo - en contraste con el desarrollo existente en países occidentales - homogeneizan y sistematizan las experiencias de diferentes grupos de mujeres, eliminando todos los modos de experiencias marginales y de resistencia (Mohanty, 2003: 68). En el caso de las mujeres de Medio Oriente, las realidades y movimientos organizados por ellas nos señalan la multiplicidad de sus experiencias de acuerdo con cada sociedad donde las mujeres no han sido sujetos pasivos. En el caso de Arabia Saudita, la resistencia abierta y vi- sible de algunas mujeres, aunque esté en sus inicios, nos muestra la lucha por una ciudadanía diferenciada que cuestiona la ciudadanía masculinizada prevaleciente en el reino saudita y la representación homogenizada de "la mujer saudita".

Como se ha analizado, la división entre esfera pública y privada en la construcción de la ciudadanía occidental no se establece tan nítidamente en la sociedad de Arabia Saudita y otras sociedades en el Medio Oriente, pues la definición de ciudadanía parte de la esfera privada al considerar a la familia y sus relaciones de parentesco como las directrices en las cuales descansa la membresía del Estado. La división entre el espacio público y privado refuerza el papel de los hombres y del Estado sobre las mujeres. La protección y la segregación reflejan la asignación de roles de acuerdo con la naturaleza femenina, por lo que las limitan al espacio privado. Los roles usualmente adscritos a las mujeres como reproductoras y trasmisoras de los valores sociales, culturales y políticos son recreados en la sociedad. Sin embargo, las mujeres activistas sin renunciar a su cultura y religión, se apropian de los símbolos y cuestionan las nociones tradicionales. De este modo, el espacio privado no ha representado necesariamente un lugar de confinamiento, algunas mujeres como las activistas han "jugado" con los límites para flexibilizarlos. Como resultado las dicotomías occidentales tradición/modernidad y esfera pública/privada, se vuelven porosas y se traspasan.

El proceso de modernización seguido por el Estado saudita no puede escapar de sus propias contradicciones. El hecho que el Estado promueva un acceso diferenciado a los recursos materiales y simbólicos no inhibe la existencia de formas de resistencia o intentos de negociación por parte de la sociedad, como se pudo ver en los diferentes momentos históricos. En el caso concreto de las mujeres en Arabia Saudita pudimos analizar cómo el Estado, al promover sus políticas de desarrollo hacia ellas, les ha otorgado de alguna manera su acceso a la esfera pública. El incremento de las mujeres que reciben 
educación y se encuentran trabajando tanto en el sector público, como en el privado, ha sido aprovechado por algunas de ellas para socializar los discursos a favor de su reconocimiento y visibilidad en la sociedad como ciudadanas. De este modo, las experiencias de las mujeres sauditas nos muestran que los agentes no son sujetos unitarios, cuyas acciones sean entendidas solamente en términos de resistencia, pues hay una ambigüedad hacia la dominación (Hollander y Einwohner, 2004: 175). Es cierto que a todo acto de dominación corresponde la resistencia, pero entre estos "dos sitios", puede haber lugar para estrategias como el acomodo y/o negociación que ayude a la misma acción de resistencia, al mismo tiempo que dicha conducta puede revestir diferentes formas y diversos momentos de acuerdo con las características estructurales y coyunturales.

En las dos coyunturas analisadas pudimos identificar las tácticas seguidas por las mujeres en cuanto a valerse del discurso oficial para reclamar sus derechos y hacerse reconocer, como durante la II guerra del Golfo, donde reclamaban un interés práctico. La táctica de la invocación de valores religiosos y sociales, así como la apropiación del discurso de defensa a favor de la mujer, les permitieron ser visibles y "dialogar" con el Estado, aunque éste se haya rehusado a otorgarles el permiso a conducir. En la segunda coyuntura, algunas mujeres habían abierto espacios que les ha permitido construir y ampliar redes para procurar cambios en su rol. Ante el debate suscitado por los acontecimientos internos y externos, algunos grupos demandaron cambios en la relación Estadosociedad. Las mujeres activistas decidieron que era el momento de levantar su propia voz y no depender de las negociaciones entre los diferentes grupos para ser incluidas. La táctica utilizada por las mujeres fue lograr la visibilidad, la utilización de los medios de comunicación, la elaboración de la petición y la participación en las elecciones, lo que ocasionó que el debate nacional se centrara en la discusión del rol de la mujer.
Este intento de algunas mujeres por hacerse visibles y demandar al Estado su reconocimiento como ciudadanas plenas nos indica que la dinámica existente entre el activismo y los derechos se mantiene en forma dialéctica, pues los derechos tienen que ser objeto de lucha, reinterpretación y defensa (Lister, Ruth, 1998:73), pese al contexto particular y no solamente en el Medio Oriente, sino en cualquier parte del mundo. Asimismo el debate de feministas radicales y postmodernas, que cuestiona las bases liberales de la ciudadanía en términos de la homogenización y abstracción de la ciudadanía, también plantea la necesidad del reconocimiento de la diferencia en la universalidad de la ciudadanía para la construcción de prácticas democráticas. En el caso de la sociedad saudita, pese a que las mujeres sauditas activistas se han constituido como parte del debate doméstico que busca concretizar a la ciudadanía, el balancear entre nociones universales que integren las diferencias, dadas las características del sistema político y cultural vigente, tiene todavía un largo recorrido.

Si en la primera coyuntura se inició un proceso de liberalización política; en la segunda con el resurgimiento de la oposición islámica radical, el gobierno tuvo que replantear sus relaciones tanto con los grupos religiosos opositores, como con los ulema oficiales y los grupos reformistas, permitiendo la flexibilización de los parámetros tradicionales. El Estado ha impulsado en años recientes bajo el gobierno del rey Abdulla una serie de cambios importantes: La celebración del primer diálogo nacional sobre la mujeres y la autorización para eliminar la necesidad de un representante legal para la mujer en el establecimiento de sus negocios, la autorización de la identificación personal de la mujer, la participación de las mujeres en las cámaras de comercio de Jedda y Damam, los cambios en las leyes de ciudadanía enunciados anteriormente, la incorporación de un grupo pequeño de mujeres a los ministerios de relaciones exteriores y la elección por parte del gobierno de cuatro mujeres como consejeras del Consejo Consultivo. Aunque estos cambios no necesariamente se traducen completamente 
en una práctica uniforme, quedan sujetos a la capacidad del gobierno de implementarlos y a sus propias necesidades de legitimidad.

La actividad de las mujeres en los años del nuevo milenio nos muestra la conformación de un movimiento de mujeres sauditas. Pese a la carencia de una organización definida con líneas de autoridad establecida, algunos sectores femeninos de las diferentes regiones del país se han unido para reclamar sus derechos. Si bien no existe un discurso unitario entre todas las mujeres sauditas, este movimiento está reelaborando las barreras tradicionales mediante su voz y presencia pública para demostrar su no conformidad con el orden social.

\section{Notas}

${ }^{1}$ La autora agradece el financiamiento otorgado por el Programa de Intercambio Sur-Sur para la investigación en la Historia del Desarrollo (SEPHIS) de Holanda y el recibimiento y asistencia del Centro de Investigación y Estudios Islámicos Rey Faisal en Riyadh, así como también a las personas entrevistadas y amistades sauditas que ayudaron en la investigación de campo. Este artículo constituye una versión revisada del trabajo presentado en el seminario Reconfigurando los Espacios de Ciudadanía: Mujeres y Género celebrado en la ciudad de México el 15 y 16 de noviembre de 2007, en el Colegio de México y la Universidad Autónoma de Méxicounidad Xochimilco.

${ }^{2}$ En el reino saudita la libertad de asociación no se establece en las regulaciones del reino, en la práctica ésta se encuentra muy limitada, pues no existen partidos políticos ni organizaciones autónomas del Estado. Las organizaciones de beneficencia y/o caritativas organizadas por mujeres y hombres existen con la aprobación del Estado y por lo general, como se mencionará posteriormente, cuentan con la participación o amparo de un miembro de la familia real.

${ }^{3}$ Las mujeres entrevistadas fueron algunas de las participantes en la manifestación de las mujeres, la elaboración de demandas, participantes en los diálogos, programas de televisión, escritoras, periodistas y mujeres de negocios. Con el fin de proteger la identidad de las entrevistas, los nombres asignados en este artículo son ficticios, solamente en caso de que alguna de nuestras entrevistadas haya publicado alguna de sus opiniones, el nombre real aparecerá.

${ }^{4}$ En occidente el término con el que se ha designado a los seguidores de este líder religioso es wahabitas, aunque ellos se hacían llamar los del llamado a la unicidad (creencia en la unicidad de Dios), ya que representaba el principio fundamental del movimiento. En la actualidad se sigue utilizando esta denominación para referirse a los religiosos sauditas, a su interpretación religiosa y al Estado.

${ }^{5}$ El sistema básico de regulaciones del reino se elabora como parte de la liberalización controlada del sistema político, ante las peticiones públicas de cambios al sistema por los diferentes grupos opositores en 1993. Al respecto véase al Rasheed, Madawi, History of Saudi Arabia, Cambridge, Cambridge University Press, 2000.

${ }^{6}$ Algunas partes de la península arábiga fueron posesiones del imperio otomano gobernadas por representantes con la cooperación de autoridades locales, especialmente las partes del norte y borde del mar rojo (hoy provincia occidental) y el golfo árabe/pérsico (provincia del Este) desde el siglo XVI, la parte central nunca fue sometida a la autoridad otomana. Al respecto véase al Rasheed, Madawi, Ibid.

${ }^{7}$ La población de Arabia Saudita está compuesta por diferentes grupos étnicos y religiosos. La división más notoria es entre la población chíta localizada principalmente al este del reino saudita y la mayoría representada por los sunnitas. Los primeros representan 10\% de la población aproximadamente. En términos étnicos hay un grupo de población negra en la ciudad de Mecca y Medina. Además, hay también una diferencia social en términos de origen tribal y aquéllos cuyo origen es más bien urbano. Ibrahim, Fouad, The shi's in Saudi Arabia, Londres, Saqui Books, 2006, pp. 55-65. 
${ }^{8}$ Mufti significa el que da opiniones legales basadas en la ley islámica y representa en el Reino la máxima autoridad religiosa. El mufti es elegido por el rey y éste a su vez elige a los miembros del Consejo Superior de los Ulema.

${ }^{9}$ Estas opiniones religiosas se pueden observar en la parte de religión de los periódicos, donde hay preguntas en específico sobre el comportamiento de la mujer entre otras cuestiones. Los ulema importantes dentro de la historia de Arabia Saudita, así como los actuales, tienen compilaciones en sus opiniones religiosas, entre ellas las que se enfocan en las mujeres.

${ }^{10}$ Sobre la educación y el énfasis sobre la importancia de la segregación véase: Doumato, Elanor,"Education in Saudi Arabia: gender, jobs and religión" en Doumato, Elanor y Pripstein, Marsha, Women and globalization in the Arab world, Londres, Lynne Rienner, pp.239-257.

${ }^{\text {" }}$ El cumplimiento de estas normas queda sujeto al grado de convicción religiosa de las personas, a los recursos económicos y a la región del país en la que se encuentre, siendo la región central y Riyad la ciudad con mayor presencia de policía religiosa.

${ }^{12}$ La educación para las mujeres, aunque se realizaba en instituciones privadas, fue mediante un decreto del rey Saud en el que se formó un comité (Presidencia General para la Educación de las Mujeres) bajo el mufti Muhmad ibn Ibrahim al-Sheik para hacerse cargo de la educación de las mujeres en 1959. Bajo esta medida modernizadora se encontraba el entonces príncipe Faisal. Hacia 1961 la primera universidad que abrió espacios para la educación superior a las mujeres en ciertas áreas como la literatura y la administración fue la Universidad de Riyad, hoy Universidad Rey Saud en la capital del reino. $\mathrm{Al}$ respecto véase $\mathrm{El}$ Hefdhy, Yahya, The Role of Ulama in establishing an Islamic education system for Saudi women, tesis de doctorado, Universidad de Florida, 1994. pp.65-75.

${ }^{13}$ En este momento, también aprovechando la coyuntura, aparecieron públicamente grupos opositores; los más contundentes y mejor organizados fueron los que representaban orientaciones religiosas como base en la propuesta por una reforma al gobierno. La separación de poderes, la independencia del poder judicial apegado a la ley islámica, la eliminación de regulaciones, la distribución de la riqueza y la apertura del sistema político fueron entre otros aspectos las críticas enunciadas por estos grupos. Al respecto véase Dekmejian, Hrair, "The raise of political islamism in Saudi Arabia" en Middle East Journal, vol. 48, núm. 4, 1994, 627-651 y Fandy, Mamoun, Saudi Arabia and the politics of dissident, Nueva York, St. Martin's Press, 1999.

${ }^{14}$ En este tiempo en términos de las leyes de costumbre no era considerado apropiado que la mujer manejara, aunque en lugares de provincia algunas mujeres lo hacían de manera discreta.

${ }^{15}$ Aunque Scott analiza la resistencia cotidiana, como formas de conducta no pública, las maneras en las que se construyen los espacios y los discursos de resistencia nos ayudan a entender las tácticas de las mujeres sauditas. Como el autor nos señala, el momento en que esta resistencia pasa a ser pública depende de las características estructurales y contingentes que rodean a los oprimidos.

${ }^{16}$ Las autoras reconocen los desacuerdos existentes en el tratamiento de la resistencia, por ello es que determinan que lo importante es identificar sus aspectos analíticos como el sentido de acción y oposición, al igual que el aspecto de la intencionalidad de los sujetos y el reconocimiento de sus acciones.

${ }^{17} \mathrm{Al}$ respecto véase Arebi, Sadeka, Women and words in Saudi Arabia: The politics of literary discourse, Nueva York, Columbia University Press, 1994

${ }^{18}$ A raíz de las demandas para el establecimiento de un Consejo Consultivo y la celebración de elecciones, las regulaciones de 1993 organizaron la autoridad del gobierno en forma vertical, donde las provincias fueron divididas en municipios. Los consejos consultivos también se formaron a nivel de provincias y a nivel municipal, los miembros fueron elegidos por el gobernador de las provincias. El príncipe heredero en el 2003 anunció las celebraciones de las elecciones municipales para elegir a la mitad de sus 
miembros. Al respecto Véase, Aba Namay, Raheed "The new Saudi representative assembly" en Islamic Law and Society, vol. 5, núm. 1, 1993, pp. 234-265.

\section{Bibliografía}

Aba Namay, Raheed, 1993, "The new Saudi representative assembly”, en Islamic Law and Society, vol. 5, núm. 1, pp. 234-265.

al-Fassi, Hatoon, 2006, "Máa nihaiat alam. Mada turidu al mar'a al saudiyya" [Hacia el fin del año. ¿Qué desea la mujer saudita?], en Iqtisadiyya, (http=www.aleqt.com/ article.php?do=show\&id=737, 20 septiembre 2006).

al-Musnad, Abdul Aziz (ed.), 1996, Islamic Fatawa Regarding Women, Riyad, Darussalam.

al-Rasheed, Madawi, 2002, A history of Saudi Arabia. Cambridge, Cambridge University Press.

Altorki, Soraya, 2000, "The concept and practice of citizenship in Saudi Arabia”, en Joseph, Suad (ed.), Gender and citizenship and the Middle East, New York: Syracuse University, pp. 215-236.

-, 1986, Women in Saudi Arabia ideology and behaviour among the elite, Nueva York, Columbia Press.

Arebi, Sadeka, 1994, Women and words in Saudi Arabia: The politics of literary discourse, Nueva York, Columbia University Press.

Aridat al nisa' al Saudiat ila Amir al Riyad [La petición de las mujeres sauditas al emir de Riyad], [n.d.p.].

Ayubi, Nazih, 1999, Overstating the Arab state: politics and society in the Middle East, Londres IB Tauris.

Chatherjee, Partha, 2006, The politics of the governed: reflections on popular politics in most of the world, New York, Syracuse University Press.

De Certau, Michel, 1996, La invención de lo cotidiano vol. 1: el arte de hacer, (traducido por Alejandro Pescador), México, Universidad Iberoamericana.

Dekmejian, Hrair, 1998, "Saudi Arabia's consultative council", en Middle East Journal vol. 52, núm. 2, pp. 216-217.

Dietz, Mary, 1994, "Ciudadanía con cara feminista: el problema con el pensamiento maternal" (traducido por Eduardo Mejía Tapia), en Debate Feminista, vol. 10, pp. 45-66.

Doumato, Eleonor, 2003, "Education, in Saudi Arabia: gender, jobs and religion”, en Doumato, Elanor and Marsha Pripstein, Women and globalization in the Arab Middle East, Londres, Lynne Rienne, pp. 239-257.

—, 1995, "The ambiguity of the shari'a and the politics of rights in Saudi Arabia”, en Afkhami, Mahnaz, Women's human rights in the Muslim world, Londres, Tauris, pp. 135-169.

El Hefdhy, Yahya, 1994, The Role of Ulama in establishing an Islamiceducation system for Saudi women, tesis de doctorado, Universidad de Florida.

Fandy, Mamoun, 1999, Saudi Arabia and the politics of dissident, Nueva York, St. Martin's Press.

Galindo, Alejandra, "Los dilemas del reino saudita", en Ruiz, Manuel (coord.), El Islamy occidente desde América Latina, México, El Colegio de México. pp. 259-288.

Hatem, Mervat, 1999, "Modernization, the state and the family in Middle East women's studies", en Meriwether, Margaret y Tucker Judith, A social history of women and gender in the modern Middle East, Boulder, Westview Press, pp. 63-88.

Ibn al Tiar, 1992, Majmu'at al-fatawi li samaht al-Sheik Abdul Aziz ibn Baz [Colección de Fatawa de su Eminencia Sheikh Abd al-Aziz ibn Baz]. Riyadh, Dar al-Watani.

Ibrahim, Fouad, 2006, The shi's in Saudi Arabia, Londres, Saqui Books.

"In defense of the nation", en Saudi Information Agency (http:www.aleqt.com/article.php?do=show\& \&id=737, english/article/cfm?qid=125\&rsid=2, 19 de junio 2004).

Joseph, Suad, 2004, "The kin contract in the Middle East", en Friedman, Marlyin (ed.), Women and Citizenship, Nueva York, Oxford Press. pp. 149-169.

Joseph, Suad (ed.), 2000, "Gendering citizenship in the Middle East", en Gender and citizenship and the Middle East, New York, Syracuse University, pp. 3-30.

Lacroix, Stéphane, 2004, "Between Islamist and liberals: Saudi Arabia's New Islamo-Liberal" Reformist", en Middle East Journal 58, 3, pp. 345-360.

50 Revista LiminaR. Estudios sociales y humanísticos, año 6, vol. VI, núm. 2, diciembre de 2008, Tuxtla Gutiérrez, Chiapas. ISSN: 1665-8027 
Lister, Ruth, "Citizenship and difference: towards a differential universalism", en European Journal of Social Theory 11, pp. 71-90.

Lughood, Lila, 2002, Feminismo y modernidad en oriente próximo [traducción de Carmen Martínez Gimeno], Madrid, Ediciones Cátedra.

Ministerio de Educación, 1980, Statistics of Higher Education 1977/78, Riyad: Minister of Higher Education.

Ministerio de Educación, 1991, Statistics of Higher Education 1989/90, Riyad: Minister of Higher Education.

Ministerio de Información.2004, Women in Saudi Arabia: care-development-improvement, Riyad, Arakan.

Ministerio del Interior, 2006, "Saudi nationality system", en (http:www.moi.gov.sa/wps/wcm/resources/file/ ebeie84274d8c95/EN_saudi_nationality-system, 24 de abril).

Mohanty, Chandra, 2003, "Under western eyes: feminist scholarship and colonial Discourses", en Feminist postcolonial theory: a reader, Edinburrgh, University of Edinburgh, pp. 49-74.

Molyneux, Marianne, 2003, Movimientos de mujeres en América Latina: estudio teórico comparado, Madrid, Ediciones Cátedra.

Movimiento para la reforma en Arabia, 1999, "Chapter 4: The truth behind the women demonstration", en http://www.miraserve.com/chap4.html, 25 de enero 1999.

"National reform document", 2004, Saudi Institute, en

(http:www.al-bab.com/arab/docs/saudi/reform2003. htm, 19 de enero 2004).

Niblock, Tim, "Social structure and the development of the Saudi Arabian political system", en Niblock, Tim (ed.), State, Society and Economy in Saudi Arabia, Londres, Croom Helm.

Piscatori, James, "The roles of Islam in Saudi Arabia's political development", en Esposito, John (ed.), Is- lam and Development, Nueva York, Syracuse University Press, pp. 123-138.

Scott, James, 2000, Los dominados y el arte de la resistencia, México, Editorial Era.

"Saudi Arabia-Constitution", 2008, en (http//www.servat.unibe.ch/icl/sa00000_html,14 de julio 2008).

"Saudi Shi' a reform demands", 2003, en Arab Press Review, 46(22), (http:www. mees. com/news/a46n22c02. htm, 9 de junio 2003).

Scott, Joan, 2001, "Gender: a useful category of historical analysis", en American Historical Review, vol. 91, núm. 51, pp. 1053-1075.

Sunder, Rajeswari, 2003, The scandal of the state: women, law and citizenship in postcolonial India, Durham y Londres, Duke University Press.

Turner, Bryan, "Islam, civil society, and citizenship", en Butenschon, Nils, Uri Davis y Manuell Hassassian, Citizenship and the state in the Middle East: approaches and applications, Nueva York, Syracuse University, pp. 28-48.

Vargas, Virginia, 1997, "Un debate feminista en curso”, en Hola, Eugenia y Portugal, Ana María, La Ciudadanía a Debate, Santiago, Chile, Isis Internacional, pp. 55-70.

Yuval-Davies, Nira (a), "Women, citizenship and difference", en Feminist Review 57, pp. 4-27.

—, 1997, "Mujeres, ciudadanía y diferencia”, en Hola, Eugenia y Portugal, Ana María, La Ciudadanía a Debate, Santiago, Chile, Isis Internacional, pp. 35-44.

Sharabi, Hisham, 1988, A theory of distorted change in Arab society, Nueva York, Oxford University Press.

\section{Fuentes Periódicas}

Al-Iqtisadiyya

Arab News

BBC Middle East News

Saudi Gazette 